\title{
Quantum Phase Transitions in Josephson-Junction Chains
}

\author{
M.-S. Choi ${ }^{1}$, J. Yi ${ }^{2}$, M.Y. Choi ${ }^{2}$, J. Choi ${ }^{3}$, and S.-I. Lee ${ }^{1}$ \\ ${ }^{1}$ Department of Physics, Pohang University of Science and Technology, Pohang 790-784, Korea \\ ${ }^{2}$ Department of Physics and Center for Theoretical Physics, Seoul National University, Seoul 151-742, Korea \\ ${ }^{3}$ Department of Physics, Keimyung University, Taegu 704-701, Korea
}

(Oct. 20 1997)

\begin{abstract}
We investigate the quantum phase transition in a one-dimensional chain of ultra-small superconducting grains, considering both the self- and junction capacitances. At zero temperature, the system is transformed into a two-dimensional system of classical vortices, where the junction capacitance introduces anisotropy in the interaction between vortices. This leads to the superconductor-insulator transition of the Berezinskii-Kosterlitz-Thouless type, as the ratios of the Josephson coupling energy to the charging energies are varied. It is found that the junction capacitance plays a role similar to that of dissipation and tends to suppress quantum fluctuations; nevertheless the insulator region survives even for arbitrarily large values of the junction capacitance.
\end{abstract}

PACS Numbers: 74.50.+r, 67.40.Db

Quantum phase transitions, which are induced by quantum fluctuations at zero temperature, are distinguished from classical phase transitions in several important respects; this has attracted much attention in recent years [1]. In particular, advances in fabrication techniques have made available arrays of ultra-small superconducting grains, where the charging energy dominates the Josephson coupling energy and accordingly, quantum fluctuation effects are of paramount importance. Such Josephson-junction arrays have become a prototype system displaying quantum phase transitions between the superconducting and the insulating phases. In the vicinity of the superconductor-insulator transition, the fluctuation effects depend crucially on the dimensionality of the system. In the case of two-dimensional (2D) arrays, rich effects of quantum fluctuations and resulting phase transitions have been examined for a rather general form of the capacitance matrix, although there still exist unsettled issues in the quantum regime, such as lowtemperature re-entrance [2 4 . On the other hand, onedimensional (1D) chains of Josephson junctions, where quantum fluctuations should be more important, have been studied mainly in the two limiting cases: the selfcharging model and the nearest-neighbor model where only nearest neighboring charges interact [5,6]. In the 1D system with only self-capacitance, the role of dissipation on the quantum phase transition [7] as well as the persistence current and voltage [8] has also been considered.

This paper investigates the quantum phase transitions in general Josephson-junction chains with both the selfand junction capacitances. At zero temperature, the system is transformed into a two-dimensional system of classical vortices, where the junction capacitance introduces anisotropy in the interaction between vortices. This leads to the superconductor-insulator transition of the Berezinskii-Kosterlitz-Thouless (BKT) type [9, 10], as the ratios of the Josephson coupling energy to the charging energies are varied. Interestingly, the junction capaci- tance here plays a role similar to that of dissipation and tends to suppress quantum fluctuations, enhancing superconductivity. However, the suppression is not strong enough, and the insulator region still remains even for arbitrarily large values of the junction capacitance.

We consider a one-dimensional array of $N$ ultra-small superconducting grains, each of which is coupled to nearest-neighboring grains via Josephson junctions. The system is characterized by three energy scales, Josephson coupling energy $E_{J}$ and charging energies $E_{0} \equiv e^{2} / 2 C_{0}$ and $E_{1} \equiv e^{2} / 2 C_{1}$, where $C_{0}$ is the self-capacitance of each grain and $C_{1}$ is the junction capacitance between nearest-neighboring grains. It is described by the Hamiltonian

$$
H=\frac{1}{2 K_{0}} \sum_{i, j=1}^{N} n_{i} C_{i j}^{-1} n_{j}-K_{0} \sum_{i=1}^{N} \cos \left(\phi_{i+1}-\phi_{i}\right),
$$

where the number $n_{i}$ of Cooper pairs and the phase $\phi_{i}$ of the superconducting order parameter at site $i$ are quantum-mechanically conjugate variables: $\left[n_{i}, \phi_{j}\right]=$ $i \delta_{i j}$, and the energy has been rescaled in units of the Josephson plasma frequency $\hbar \omega_{p} \equiv \sqrt{8 E_{0} E_{J}}$. Here we have introduced $K_{0} \equiv \sqrt{E_{J} / 8 E_{0}}$ to describe the case $C_{0} \neq 0$, and written the capacitance matrix in the form

$$
C_{i j}=\delta_{i j}+\lambda^{2}\left(2 \delta_{i, j}-\delta_{i, j-1}-\delta_{i, j+1}\right),
$$

where $\lambda^{2} \equiv C_{1} / C_{0}$. On the other hand, when $C_{0}=0$, it is convenient to introduce $K_{1} \equiv \sqrt{E_{J} / 8 E_{1}}$. Note that $\lambda$ can also be written in the form: $\lambda=K_{1} / K_{0}$.

In the imaginary-time path-integral representation, the partition function of the system reads

$$
Z=\prod_{j, \tau} \sum_{n_{j, \tau}} \int_{0}^{2 \pi} \frac{d \phi_{j, \tau}}{2 \pi} \exp \left\{-\sum_{\tau=0}^{\beta-1} L[n, \phi]\right\}
$$

with the action 


$$
L[n, \phi]=i \sum_{j}^{N} n_{j, \tau} \partial_{\tau} \phi_{j, \tau}+\frac{1}{2 K_{0}} \sum_{i, j}^{N} n_{i, \tau} C_{i j}^{-1} n_{j, \tau}-K_{0} \sum_{j}^{N} \cos \partial_{x} \phi_{j, \tau},
$$

where the temperature has been rescaled according to $\hbar \omega_{p} \beta \rightarrow \beta$. In Eq. (3), $\partial_{x}$ and $\partial_{\tau}$ denote the difference operator with respect to the position $x$ and to the imaginary time $\tau$, respectively: $\partial_{x} \phi_{j, \tau} \equiv \phi_{j+1, \tau}-\phi_{j, \tau}$ and $\partial_{\tau} \phi_{j, \tau} \equiv \phi_{j, \tau+1}-\phi_{j, \tau}$, and the (imaginary) time slice $\delta \tau$ in the interval $[0, \beta]$ has been chosen to be unity (in units of $\hbar \omega_{p}$ ) [11. Our main concern here is the quantum phase transition at zero temperature, and accordingly, the limit $\beta \rightarrow \infty$ as well as the thermodynamic limit $N \rightarrow \infty$ is to be taken. We then apply the Villain approximation 12] and integrate out $\left\{\phi_{j, \tau}\right\}$, to obtain the partition function in terms of an additional set of integer variables $\left\{m_{j, \tau}\right\}$ (as well as $\left\{n_{j, \tau}\right\}$ ). The corresponding action is given by

$$
L[n, m]=\frac{1}{2 K_{0}} \sum_{i, j} n_{i, \tau} C_{i j}^{-1} n_{j, \tau}+\frac{1}{2 K_{0}} \sum_{j} m_{j, \tau}^{2}
$$

where the two sets of integer variables satisfy the constraint $\partial_{x} m_{j, \tau}+\partial_{\tau} n_{j, \tau}=0$. This constraint is conveniently taken into account by introducing an integer field $A_{\tilde{j}, \tilde{\tau}}$ defined on the space-time dual lattice $(\tilde{j}, \tilde{\tau}) \equiv$ $(j+1 / 2, \tau+1 / 2)$ in such a way that $m_{j, \tau}=-\partial_{\tau} A_{\tilde{j}, \tilde{\tau}}$ and $n_{j, \tau}=\partial_{x} A_{\tilde{j}, \tilde{\tau}}$. Henceforth we will work on the dual lattice, and drop for simplicity the tilde sign over site indices. The partition function is thus written in terms of the unconstrained summation of $\exp \left\{-\sum_{\tau} L[A]\right\}$ over $A_{j, \tau}$ 's, with the action

$$
L[A]=\frac{1}{2 K_{0}} \sum_{i, j}\left(\partial_{x} A_{j, \tau}\right) C_{i j}^{-1}\left(\partial_{x} A_{j, \tau}\right)+\frac{1}{2 K_{0}} \sum_{j}\left(\partial_{\tau} A_{j, \tau}\right)^{2} .
$$

Finally, the Poisson summation formula, which decomposes $A_{j, \tau}$ into a real-valued field and a new integer field $v_{j, \tau}$, allows us to integrate out the real-valued field $A_{j, \tau}$. This leads, apart from the spin-wave part, to the 2D system of classical vortices described by the Hamiltonian

$$
H_{v}=2 \pi^{2} K_{0} \sum_{i \tau, j \sigma} v_{i, \tau} U(i-j, \tau-\sigma) v_{j, \sigma}
$$

where the vortex interaction is given by

$$
\begin{aligned}
& U(x, \tau)=\int_{0}^{2 \pi} \frac{d q}{2 \pi} \int_{0}^{2 \pi} \frac{d \omega}{2 \pi} e^{+i q x-i \omega \tau} \widetilde{U}(q, \omega), \\
& \widetilde{U}(q, \omega)=\left[\frac{\Delta(q)}{\widetilde{C}(q)}+\Delta(\omega)\right]^{-1}
\end{aligned}
$$

with the Fourier transforms of the lattice Laplacian $\Delta(z)=2(1-\cos z)$ and of the capacitance $\widetilde{C}(q)=$ $1+\lambda^{2} \Delta(q)$. Note that unless $C_{0}=0$, the diagonal piece $U_{0} \equiv U(x=0, \tau=0)$ becomes arbitrarily large as $N$ and $\beta$ are increased to infinity. Therefore the diagonal term $2 \pi^{2} K_{0} U_{0}\left(\sum_{j, \tau} v_{j, \tau}\right)^{2}$ should vanish in Eq. (6), leaving the vorticity neutrality condition: $\sum_{j, \tau} v_{j, \tau}=0$, and the Hamiltonian (6) may be written in the form

$$
H_{v}=-\pi K_{0} \sum_{i \tau, j \sigma} v_{i, \tau} \widehat{U}(i-j, \tau-\sigma) v_{j, \sigma}
$$

with $\widehat{U}(x, \tau) \equiv 2 \pi\left[U_{0}-U(x, \tau)\right]$. The behavior of the (reduced) vortex interaction $\widehat{U}(x, \tau)$ for several values of $\lambda$ is displayed in Figs. 11 and 2, which manifests the logarithmic behavior at large length scales. In particular, the short-range anisotropy prominent for large values of $\lambda$ decreases rapidly with the distance. According to the renormalization group (RG) theory of the $2 \mathrm{D}$ Coulomb gas, such short-range anisotropy should not affect the universality class. Thus in the spirit of the RG theory, the system is expected to exhibit qualitatively the same critical behavior as the 2D Coulomb gas [13].

We now investigate the quantum phase transitions displayed by the Hamiltonian in Eq. (6). First we consider the simplest case of $C_{1}=0$, which has been studied in Ref. 15. In this self-charging limit, the interaction in

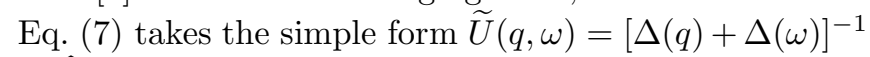
or $\widehat{U}(x, \tau) \approx \ln \sqrt{x^{2}+\tau^{2}}+\frac{3}{2} \ln 2+\gamma_{E}$, where $\gamma_{E}$ is the Euler number. Thus the Hamiltonian (\$) describes precisely the isotropic 2D Coulomb gas, and the system undergoes a BKT transition [9, 10, 12] from the insulating phase to the superconducting one as $K_{0}$ is increased [5].

We next consider the opposite limit $C_{0}=0$, where the relevant (dimensionless) coupling constant is $K_{1}$ instead of $K_{0}$. Accordingly, it is proper to use $\hbar \omega_{p} \equiv \sqrt{8 E_{1} E_{J}}$ in rescaling the energy and the imaginary time, which in turn gives $\widetilde{C}(q)=\lambda^{-2}+\Delta(q)$ and $\widetilde{U}(q, \omega)=[1+\Delta(\omega)]^{-1}$. As a result, we obtain the Hamiltonian in the form

$$
H_{v}=2 \pi^{2} K_{1} \sum_{i \tau, j \sigma} v_{i, \tau} U(i-j, \tau-\sigma) v_{j, \sigma}
$$


where, in sharp contrast to the previous self-charging case, the vortex interaction $\widehat{U}(x, \tau) \equiv 2 \pi\left[U_{0}-U(x, \tau)\right]$ is short-ranged:

$$
\widehat{U}(x, \tau) \approx \frac{2 \pi}{\sqrt{5}}\left[1-\delta_{x, 0} e^{-|\tau|}\right]
$$

for $|\tau| \gg 1$. It is further of particular importance that $U_{0}$ in general does not diverge, which implies that the diagonal term in the Hamiltonian (9) does not give the vorticity neutrality condition. Thus (unbound) free vortices become pervasive, and the system remains insulating for any nonzero value of $K_{1}$. This can also be understood in the charge representation, where the partition function reads

$$
Z=\prod_{j, \tau} \sum_{n_{j, \tau}} \exp \left\{-\frac{1}{\beta N} \sum_{q, \omega}|n(q, \omega)|^{2} G^{-1}(q, \omega)\right\},
$$

with $G(q, \omega)=\Delta(q)[\Delta(\omega)+1]^{-1}$. It follows from the analytic continuation of $G(q, \omega)$ that the charge excitation has a gap $E_{g} \sim \hbar \omega_{p}$. Thus the long-range interaction $C_{i j}^{-1}$ between charges gives rise to the gap, resulting in an insulator. It is also of interest to compare this case with the "nearest-neighbor model" in Ref. [5]: In the latter, only the nearest-neighboring charges interact, which allows only bound vortices. Consequently, the system is superconducting for all finite values of the interaction strength.

We now turn to the more realistic case that $\lambda$ is small but nonzero, i.e., $C_{0} \gg C_{1}$. In this case, the vortex interaction $\widehat{U}$ is, to the order of $\lambda^{2}$, isotropic and has the asymptotic behavior: $\widehat{U}(x, \tau) \approx \ln \sqrt{x^{2}+\tau^{2}}+\varepsilon(\lambda)$, where $\varepsilon(\lambda)$ is the vortex pair creation energy (per vortex) given by $\varepsilon(\lambda) \approx(3 / 2) \ln 2+\gamma_{E}+(\pi-1) \lambda^{2}+\mathcal{O}\left(\lambda^{4}\right)$. Hence the system again reduces to the $2 \mathrm{D}$ Coulomb gas, with the vortex fugacity $y(\lambda) \equiv \exp \left[-2 \pi K_{0} \varepsilon(\lambda)\right]$ diminished by the factor $\exp \left[-2 \pi K_{0}(\pi-1) \lambda^{2}\right]$. The standard RG theory of the BKT transition [10,12 then predicts the transition point $K_{0}^{c}(\lambda)$ slightly decreased from $K_{0}^{c}(0)$ by the amount

$$
\frac{\delta K_{0}^{c}}{K_{0}^{c}(0)}=-(\pi-1) \frac{2 \pi K_{0}^{c}(0)-4}{2 \pi K_{0}^{c}(0)-3} \lambda^{2}+\mathcal{O}\left(\lambda^{4}\right) \approx-1.6 \lambda^{2} .
$$

In this limit $(\lambda \ll 1)$, the Hamiltonian in Eq. (11) can also be represented in terms of phase variables, which yields a $2 \mathrm{D} \mathrm{XY}$ model with an additional interaction for nonzero $\lambda$. To see this, we neglect $\mathcal{O}\left(\lambda^{4}\right)$, and write the inverse capacitance matrix in the form

$$
C_{i j}^{-1} \approx\left(1-2 \lambda^{2}\right) \delta_{i j}+\lambda^{2}\left(\delta_{i, j+1}+\delta_{i, j-1}\right) .
$$

Note that without the off-diagonal term in $C_{i j}^{-1}$, the first and the second terms in the action (3) would just be the Villain form of the cosine action along the $\tau$ direction [5.87. To examine the effects of the off-diagonal term, we use the identity

$$
\exp \left[\frac{\lambda^{2}}{4 K_{0}}\left(\partial_{x} n_{j, \tau}\right)^{2}\right]=\frac{1}{\sqrt{\pi}} \int_{-\infty}^{\infty} d z_{j, \tau} \exp \left[-z_{j, \tau}^{2}-\frac{\lambda}{\sqrt{K_{0}}} z_{j, \tau} \partial_{x} n_{j, \tau}\right]
$$

which allows to separate the charge variables at different sites. The resulting action then takes the Villain form of the cosine action with the argument $\partial_{\tau} \phi_{j, \tau}+i \sqrt{\lambda^{2} / K_{0}} \partial_{x} z_{j, \tau}$ along the $\tau$ direction. To the order of $\lambda^{2}$, the Gaussian integration over $z_{j, \tau}$ leads to the effective phase Hamiltonian

$$
\begin{aligned}
H_{\mathrm{eff}}[\phi]= & -K_{0}\left(\frac{1}{\sqrt{1-\lambda^{2}}}+\frac{\lambda^{2}}{4 K_{0}}\right) \sum_{j, \tau}\left[\cos \left(\partial_{x} \phi_{j, \tau}\right)+\cos \left(\partial_{\tau} \phi_{j, \tau}\right)\right] \\
& +\lambda^{2} K_{0} \sum_{j, \tau}\left[\partial_{\tau} \sin \partial_{x} \phi_{j, \tau}\right]^{2}
\end{aligned}
$$

where the anisotropy has been removed by rescaling again the $\tau$ axis by the factor $\left(1-\lambda^{2}\right)^{-1 / 2}+\lambda^{2} / 4 K_{0}$. Note that Eq. (14) reduces, for $\lambda=0$, to the standard $2 \mathrm{D} \mathrm{XY}$ Hamiltonian. Here the junction capacitance not only enhances the effective coupling of the XY model but also introduces an additional interaction given by the second term in Eq. (14). Interestingly, the latter is very similar in form to the dissipation term in the effective action, which is known to suppress quantum fluctuations [3, 7]. Therefore both effects contribute to the enhancement of 
phase coherence, and it is concluded that the junction capacitance in general tends to suppress quantum fluctuations induced by the self-capacitance, thus reducing the insulator region.

For larger values of $\lambda$, Figs. 1 and 2(b) show that the vortex creation energy $\varepsilon(\lambda)$ and the short-range behavior of the interaction $\widehat{U}(x, \tau)$ are highly anisotropic. Still at large length/time scales, $\widehat{U}(x, \tau)$ becomes isotropic and logarithmic, which can also be confirmed by the asymptotic expansion for large $\tau$ :

$$
\widehat{U}(0, \tau)=\frac{2 \pi}{\sqrt{5}} \lambda+\ln \tau+\left\{\gamma_{E}+2 \ln 2-\frac{1}{2} \ln 5-\frac{127}{150}\right\}+\mathcal{O}\left(1 / \tau, 1 / \lambda^{2}\right) .
$$

Although the anisotropy in the short-range behavior of $\widehat{U}$ may slightly alter the details of the RG flow, the qualitative features at large length scales are expected unaffected. Thus it is concluded that for any value of finite $\lambda$ the system undergoes the superconductor-insulator transition of the BKT universality class.

Figure 3 displays the schematic phase diagram on the $K_{0}-K_{1}$ plane. Although the precise phase boundary, i.e., the detailed behavior of $K_{0}^{c}(\lambda)$, in general depends on the microscopic length scales such as the (imaginary) time slice $\delta \tau$, the universal (large-length scale) behavior should not be affected. Thus the phase boundary between the superconducting and the insulating phases is concluded to belong to the BKT universality class. As $\lambda\left(\equiv K_{1} / K_{0}\right)$ is increased, the vortex creation energy also grows monotonically, resulting in the decrease of $K_{0}^{c}(\lambda)$ toward the limiting value $2 / \pi$. (Note that the value $2 / \pi$ corresponds to the zero vortex fugacity or the infinite vortex creation energy.) This lowering of the transition point and the resulting enhancement of superconductivity reflects the role of the junction capacitance: It tends to suppress the quantum fluctuations induced by the selfcapacitance, as manifested in the phase representation given by Eq. (14). There has been few experimental studies of quantum phase transitions in 1D Josephson jucntion arrays. Recent interest in 1D tunnel junction arrays [14 and advances in submicron fabrication techniques strongly motivate experimenters in this field.

MSC is grateful to W. G. Choe, G. S. Jeon, and K.-H. Wagenblast for helpful discussions. This work was supported in part by the Basic Science Research Institute Program, Ministry of Education of Korea and in part by the Korea Science and Engineering Foundation through the SRC Program.

[1] See, e.g., S. L. Sondhi, S. M. Girvin, J. P. Carini, and D. Shahar, Rev. Mod. Phys. 69, 315 (1997) and reference therein.
[2] R. Fazio and G. Schön, Phys. Rev. B 43, 5307 (1991); A. van Otterlo, K.-H. Wagenblast, R. Fazio, and G. Schön, ibid. 48, 3316 (1993).

[3] B. J. Kim and M. Y. Choi, Phys. Rev. B 52 (5), 3624 (1995); ibid. 56, 395 (1997).

[4] C. Rojas and J. V. José, Phys. Rev. B 54, 12361 (1996).

[5] R. M. Bradley and S. Doniach, Phys. Rev. B 30, 1138 (1984).

[6] It should be noted that the second case (the nearestneighbor model in Ref. [5]) is rather unrealistic and in particular does not correspond to the case $C_{0}=0$ and $C_{1} \neq 0$ shown schematically in Fig. 2 of Ref. [5]. In the latter case, the charge interaction $C_{i j}^{-1}$ is long-ranged rather than between nearest neighbors.

[7] P. A. Bobbert, R. Fazio, G. Schön, and G. T. Zimanyi, Phys. Rev. B 41, 4009 (1990); P. A. Bobbert, R. Fazio, G. Schön, and A. D. Zaikin, ibid. 45, 2294 (1992).

[8] M. Y. Choi, Phys. Rev. B 48, 15920 (1993).

[9] V. L. Berezinskii, Zh. Eksp. Teor. Fiz. 59, 907 (1970) [Sov. Phys. JETP 32, 493 (1971)]; J. M. Kosterlitz and D. J. Thouless, J. Phys. C 6, 1181 (1973).

[10] J. M. Kosterlitz, J. Phys. C 7, 1047 (1974).

[11] The critical behavior of the system should not be affected by the choice of $\delta \tau$ (see Ref. (1, in). Here all the dynamics of the system occurs over the time scale $1 / \omega_{p}$, making it proper to choose $\delta \tau \sim \hbar \omega_{p}$.

[12] J. V. José, L. P. Kadanoff, S. Kirkpatrick, and D. R. Nelson, Phys. Rev. B 16, 1217 (1977).

[13] P. Minnhagen, Rev. Mod. Phys. 59, 1001 (1987).

[14] See, e.g., P. Delsing, D. B. Haviland, and P. Davidsson, Czech. J. Phys. 46, 2359 (1996), and reference therein.

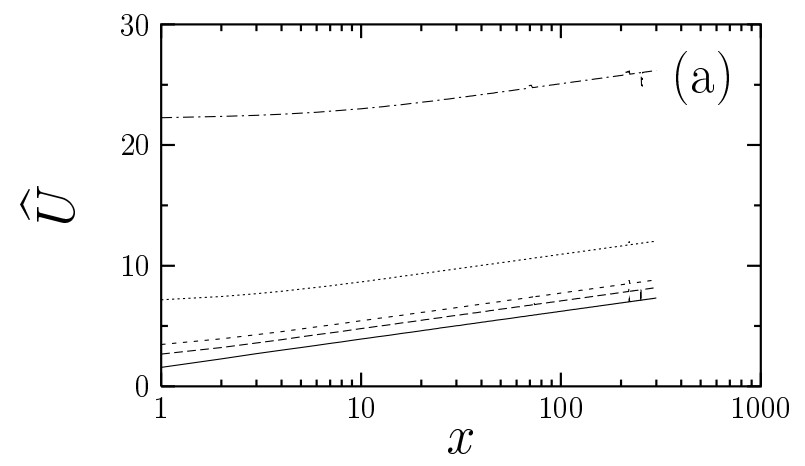




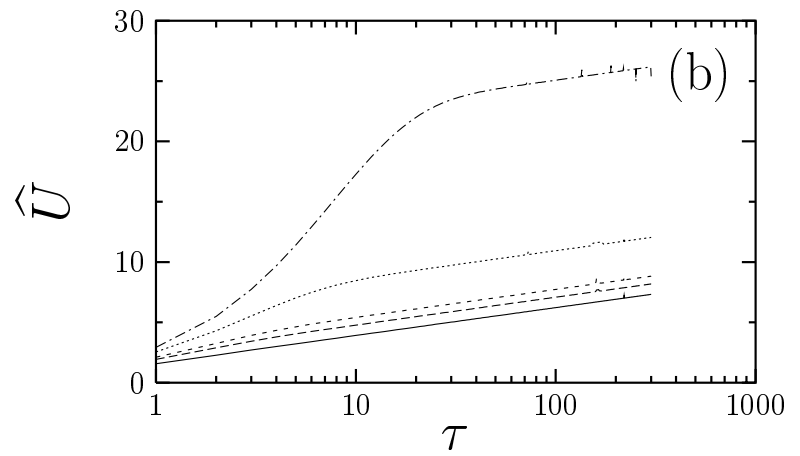

FIG. 1. Vortex interaction potential $\widehat{U}$ along (a) the $x$-axis and (b) the $\tau$-axis for $\lambda=0,0.5,1,5$, and 50 (from the bottom to the top).

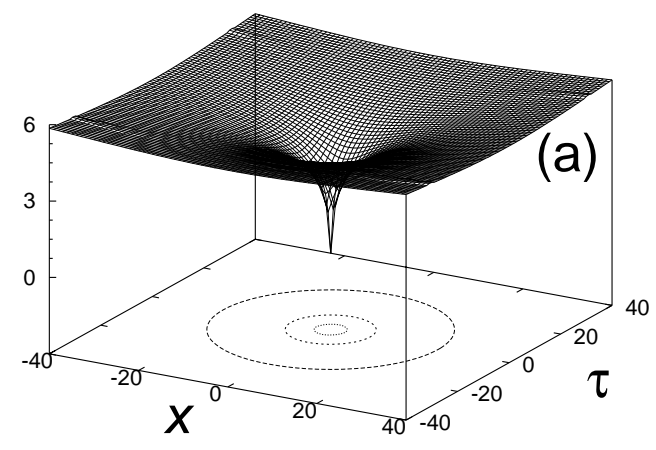

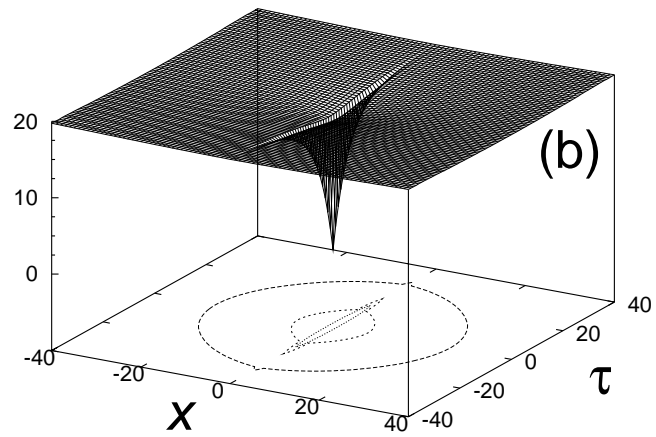

FIG. 2. Plot of the vortex interaction $\widehat{U}(x, \tau)$ for (a) $\lambda^{2}=0.1$ and (b) $\lambda^{2}=30$.

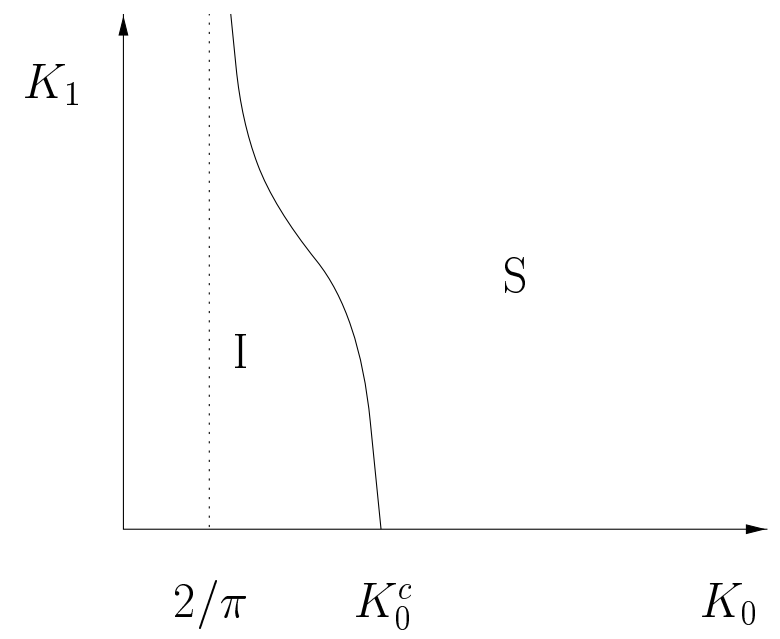

FIG. 3. Schematic phase diagram of a Josephson-junction chain at zero temperature. As $\lambda$ gets increased, $K_{0}^{c}$ decreases toward the limiting value $2 / \pi$. 